\title{
Etoricoxib-induced life-threatening hyperkalemia and acute kidney dysfunction against the background of telmisartan and a low sodium diet
}

\author{
Swagata Tripathy $\cdot$ Suresh Chandra Dash
}

Received: 17 January 2010 / Accepted: 15 June 2010 /Published online: 20 August 2010

(C) Springer-Verlag London Ltd 2010

\begin{abstract}
Drug-induced hyperkalemia is not uncommon and may be life-threatening when presenting acutely in the emergency department. We present a case of severe hyperkalemia precipitated acutely by etoricoxib in a patient who was on telmisartan and a low sodium (potassium chloriderich) diet. A 75-year-old male with a past medical history of well-controlled diabetes and hypertension was prescribed etoricoxib (90 mg daily) for 3 days for musculoskeletal backache. He had been taking his routine medications including telmisartan and a potassium-rich salt substitute for many years, without any recent change in dosage or quantity. There was evidence of microalbuminurea; however, the renal functions and electrolytes prior to starting etoricoxib were normal. He presented to the emergency department with signs and symptoms of lifethreatening hyperkalemia (serum potassium $7.7 \mathrm{mEq} / \mathrm{dl}$ ), accelerated hypertension, congestive heart failure, pulmonary edema and acute renal failure. Acute medical management and withholding all drugs that could cause hyperkalemia improved his serum potassium levels over 24 $\mathrm{h}$ and renal parameters within 5 days. All the other drugs except etoricoxib were restarted under observation over 8 weeks with no recurrence of the acute episode. Nonsteroidal analgesics and other COX-2 inhibitors (rofecoxib and celecoxib) have been known to precipitate renal failure
\end{abstract}

\footnotetext{
S. Tripathy $(\square)$

Department of Anaesthesia, Kalinga Institute of Medical Sciences, Bhubaneswar, India

e-mail: tripathyswagata@gmail.com

S. C. Dash

Department Nephrology, Kalinga Institute of Medical Sciences, Bhubaneswar, India

e-mail: scdash@gmail.com
}

and hyperkalemia specially in patients at risk for the same; although not unexpected, this may be the first reported case of life-threatening hyperkalemia precipitated by etoricoxib in a previously stable patient having increased risk of renal failure and hyperkalemia.

Keywords Hyperkalemia $\cdot$ Renal failure $\cdot$ Etoricoxib · NSAIDs · COX2 inhibitors

\section{Background}

Medications have been cited as a primary or contributing cause of hyperkalemia in $35 \%$ to $75 \%$ of hospitalized patients [1]. Nonsteroidal anti-inflammatory drugs (NSAIDs) may be associated with more than $10 \%$ of these cases [2]. COX-2 inhibitors appear to have similar effects to NSAIDS via the inhibition of renal prostaglandin synthesis [3]. The susceptibility to drug-induced hyperkalemia is higher in patients with impaired renal function, diabetes mellitus and states of impaired potassium homeostasis such as hypoaldosteronism [4].

\section{Case report}

A 75-year-old male patient presented to the emergency department in acute respiratory distress. The patient had a history of diabetes, hypertension and hyperthyroidism lasting for several years. His blood pressure, blood sugar (including $\mathrm{Hb} 1 \mathrm{Ac}$ ) and thyroid status were well controlled with medicines (Table 1). For the last 5 years, he had been on a 'low salt' diet under medical advice. The 'light salt' diet is primarily composed of potassium chloride salt 
Table 1 Patient medications

\begin{tabular}{llll}
\hline Medications & Dose & Route & Frequency \\
\hline Nifedipine & $20 \mathrm{mg}$ & Orally & Twice a day \\
$\begin{array}{l}\text { Telmisartan/ } \\
\text { hydrochlorthiazide }\end{array}$ & $40 \mathrm{mg}$ & Oral & Once a day \\
Torsemide & $10 \mathrm{mg}$ & Oral & Once a day \\
Atorvastatin & $10 \mathrm{mg}$ & Oral & Once a day \\
Ecospirin & $75 \mathrm{mg}$ & Oral & Once a day \\
Levothyroxine & $50 \mu \mathrm{g}$ & Oral & Once a day \\
Insulin mixtard (30:70) & $34 \mathrm{U} / 24 \mathrm{U}$ & Subcutaneous & \\
& & &
\end{tabular}

(approximate consumption $3.5 \mathrm{~g} / \mathrm{day}$ ). There were no recent changes in his diet or medications. He had not been on any herbal medications, potassium supplements, potassium-sparing diuretics or over-the-counter analgesics. He had documented evidence of diabetic neuropathy and microalbuminurea (normal creatinine clearance by the Cockroft-Gault formula), which had not been deteriorating up to his last routine examination 1 month prior to admission (Table 2). An electrocardiogram done 15 days prior to this emergency admission did not show any changes suggestive of hyperkalemia such as tall $t$ waves, increased PR interval or broad QRS complex.

The patient was treated 5 days prior to admission for low backache of musculoskeletal origin by his regular physician who prescribed etoricoxib $90 \mathrm{mg} /$ day for 3 days. He had taken the last dose $48 \mathrm{~h}$ prior to presenting to us. He had complaints of nausea, weakness, lassitude, swelling of the legs and dyspnea for the last $72 \mathrm{~h}$ with gradually increasing respiratory distress.

On presentation our patient was afebrile, with a pulse rate of $60 / \mathrm{min}$, blood pressure $180 / 90 \mathrm{mmHg}$, respiratory rate 30 per minute and oxygen saturation of $82 \%$ on room air. Examination showed a moist tongue, bilateral pitting pedal edema, raised jugular venous pressure and crepitations at the lung bases bilaterally.

Oxygen inhalation and $40 \mathrm{mg}$ intravenous furosemide were given, and the patient was shifted to the intensive care unit promptly as his restlessness increased. The electrocardiogram done here showed a heart rate of $38 / \mathrm{min}$ with a near sine wave pattern that was broad QRS complex, with absent $\mathrm{p}$ waves and tall tented T waves in lead II (Fig. 1).

Aggressive medical management of hyperkalemia (revealed in the blood gas electrolyte study) with $10 \mathrm{ml}$ of $10 \%$ calcium gluconate, hourly nebulization with salbutamol and glucose with insulin infusion administered intravenously (25\% dextrose, $100 \mathrm{ml}$, with 10 units of regular insulin) reverted the electrocardiogram changes (Fig. 2) and decreased the serum potassium level after $8 \mathrm{~h}$ to $6.5 \mathrm{meq} / \mathrm{dl}$. Telmisartan, torsemide, hydrochlorthiazide and light salt were discontinued. Parenteral furesemide (40 mg twice a day), tramadol (an opioid analgesic) for pain relief and the addition of salt to the diet were instituted. Respiratory status improved, and pedal edema resolved within $24 \mathrm{~h}$. Biochemical parameters (Table 2) revealed life-threatening hyperkalemia, deranged renal function and hyponatremia. Complete blood count with a differential, serum-free T3, T4, TSH and lipid profile was within normal limits. Urinalysis on admission showed a $\mathrm{pH}$ of 5.0 , specific gravity $1.005,2+$ protein, $2+$ glucose, negative ketones and no microbiological growth on culture. Urine sediment showed few epithelial cells and granular casts. Urine sodium concentration was $96 \mathrm{mEq} / \mathrm{l}$. The chest radiograph showed cardiomegaly and cardiogenic pulmonary edema.

Echocardiogram showed a left ventricular hypertrophy with a good left ventricular systolic function. Renal ultrasonogram showed no evidence of hydronephrosis, significant prostatomegaly or other masses. On fundus examination, posterior segment details were unremarkable.

There was a return of serum potassium to normal levels within $24 \mathrm{~h}$. Serum sodium gradually improved over 6 days to $130 \mathrm{meq} / \mathrm{dl}$. Serum urea decreased to pre-admission values by day 5 when he was discharged. The case was discussed with his primary physician, and with the consent of the patient, telmisartan and a low salt supplement were carefully restarted one at a time. On follow-up at 2 months, he had restarted all his previous medications including telmisartan, and his electrolyte and renal parameters were at his usual baseline levels.

\section{Discussion}

The NSAID etoricoxib is a selective inhibitor of cyclooxygenase 2 (COX-2) approved for treatment of patients with chronic arthropathies and musculoskeletal and dental pain [5]. Like parecoxib, celecoxib and other selective COX-2 inhibitors, it has demonstrated a significant reduction in gastrointestinal toxicity, although its renal adverse effects appear to be similar to those of other NSAIDs. The
Table 2 Biochemical parameters

-3 M: 3 months prior to admission, $-1 \mathrm{M}$ : 1 month prior to admission, D: day

\begin{tabular}{llllllll}
\hline & $-3 \mathrm{M}$ & $-1 \mathrm{M}$ & $\mathrm{D} 1$ & $\mathrm{D} 2$ & $\mathrm{D} 3$ & $\mathrm{D} 4$ & $\mathrm{D} 5$ \\
\hline Serum urea $(12-25 \mathrm{mg} / \mathrm{dl})$ & 38 & 42 & 62 & 78 & 63 & 52 & 45 \\
Serum creatinine(0.5-1.2 $\mathrm{mg} / \mathrm{dl})$ & 1.3 & 1.2 & 1.8 & 2.3 & 1.9 & 1.5 & 1.2 \\
Serum sodium $(136-145 \mathrm{meq} / \mathrm{dl})$ & - & 132 & 103 & 109 & 117 & 123 & 130 \\
Serum Potassium $(3.5-5.3 \mathrm{meq} / \mathrm{dl})$ & - & 3.8 & 7.7 & 4.7 & 3.6 & 3.8 & 3.5 \\
\hline
\end{tabular}




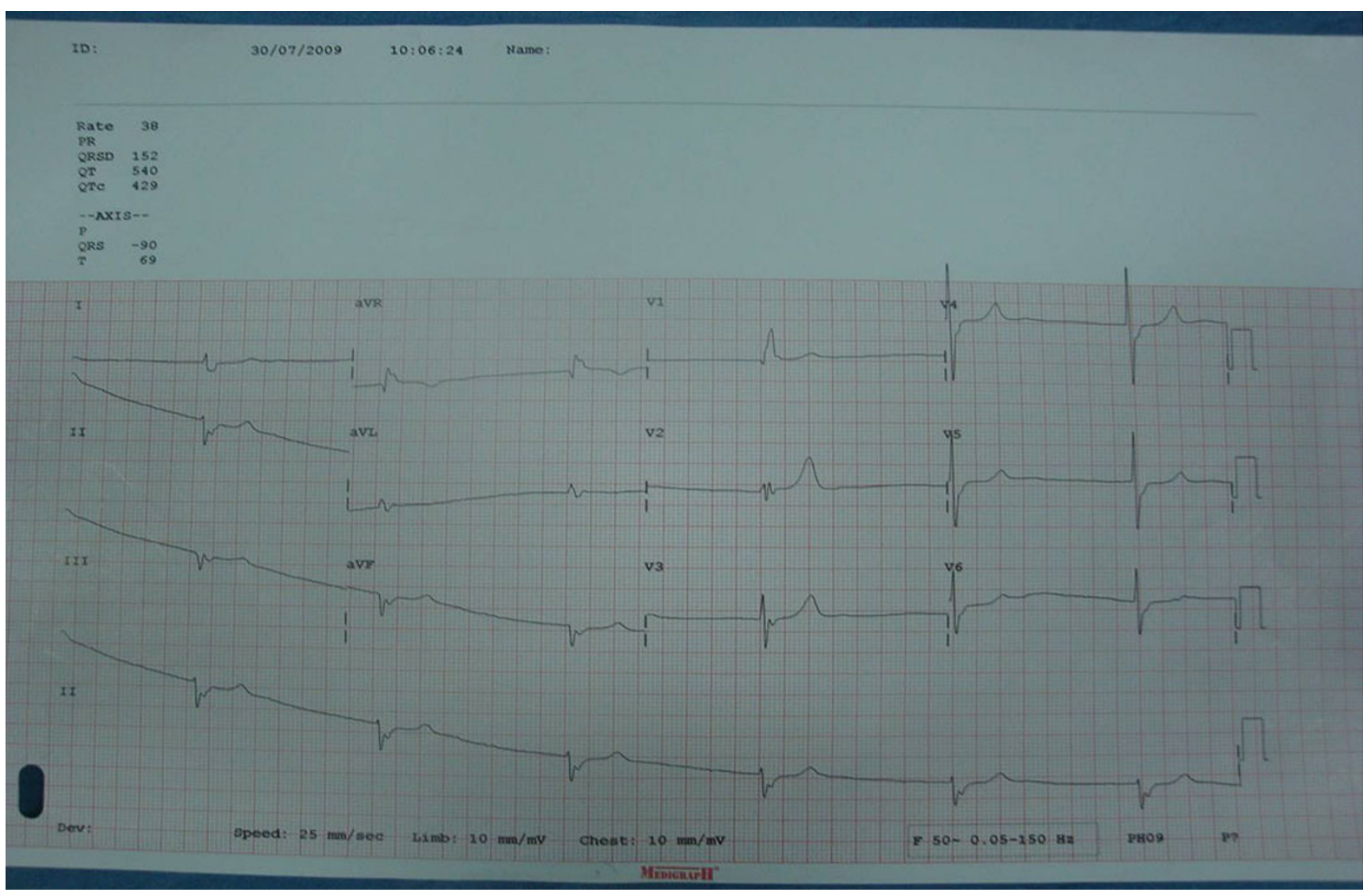

Fig. 1 The initial 12-lead ECG $(25 \mathrm{~mm} / \mathrm{s}, 5 \mathrm{~mm} / \mathrm{mV})$ obtained on presentation to the emergency department demonstrates a sinus bradycardia with prolonged atrial conduction (flattened $\mathrm{P}$ waves) and an intraventricular conduction delay (QRS, $154 \mathrm{~ms})$

cardiovascular adverse effects of the selective COX-2 inhibitors like myocardial infarction, hypertension, fluid retention and congestive heart failure, among others, have led to a revoking of Merck's Vioxx (rofecoxib) and the

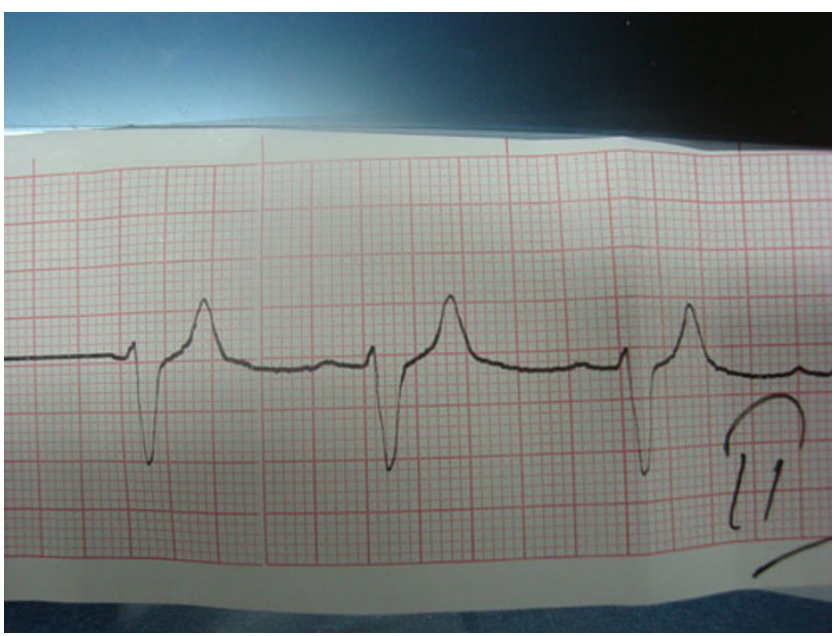

Fig. 2 Lead II of the ECG done after an hour of initiation of medical management showing improvement of heart rate and reappearance of the $\mathrm{p}$ waves unavailability of Arcoxia (etoricoxib) in the USA [6, 7]. They are widely prescribed in Europe, South America and Asia for musculoskeletal pain. They are preferred over other NSAIDS mainly due to the favorable gastrointestinal profile and convenience of single-day dosing. It is available as an offthe-counter drug in India.

Specific COX-2 inhibition may induce renal ischemia, electrolyte imbalance and increased blood pressure, ultimately leading to fluid and sodium retention and decreased GFR [8]. In elderly patients on a sodium-depleted diet, a drop in GFR may be significant after a single dose of COX2 inhibitor [9].

In our case, acute renal insult and inhibition of prostaglan$\operatorname{din} \mathrm{I}_{2}$ (which increases potassium secretion) as a result of COX-2 inhibition is the proposed mechanism precipitating hyperkalemia in our patient who was already on 'low salt' supplements rich in potassium and telmisartan, which causes hyperkalemia by inducing a state of hypoaldosteronism [10]. The time course of events, absence of other precipitating causes of renal dysfunction or hyperkalemia, rapid improvement on stopping the drug with no recurrence on reintroduction of the other medications and diet make etoricoxib the most probable cause (Naranjo score 7). 
Similar to non-selective NSAIDS, selective COX-2 inhibition may cause edema, hypertension and acute renal failure in a minority of patients. It may exacerbate preexisting hypertension. The number and spectrum of these side effects have been greater with rofecoxib than with the other COX-2 inhibitors, presumably due to the non-class effects of these drugs as their chemical structures are different [11].

In 14 cases of COX-2 inhibitor (celecoxib and rofecoxib) associated acute renal failure reported by Perazella et al. [12], all the patients had several risk factors for NSAIDinduced nephrotoxicity, including renal compromise and multiple medications. Acute renal failure, disturbances in volume status (heart failure, edema), acidosis and hyponatremia were common. Twelve patients developed hyperkalemia $(>5.1 \mathrm{meq} / \mathrm{dl})$ and four required hemodialysis. Similar to our case, treatment before the development of clinically recognized renal failure ranged from 4 days to 3 weeks, and resolution of the renal dysfunction took 2 days to 3 weeks after discontinuation of drugs and supportive therapy.

More specifically for etoricoxib, recent data from the large MEDAL (Multinational Etoricoxib vs. Diclofenac Arthritis Long Term) program, which enrolled 34,000 arthritis patients over 18 months, show clinically important increases in renal function endpoints. There was no significant increase in the incidence of hyperkalemia [13].

Unlike in healthy people, patients with edematous conditions requiring dietary sodium restriction and diuretics, such as those with chronic renal insufficiency, congestive heart failure, chronic liver disease and hypertension, should be treated cautiously with COX-2 inhibitors. Electrolytes and renal function should be checked 1-2 weeks after initiation of COX-2 inhibitors in these patients to screen for hyperkalemia and renal insufficiency $[14,15]$.

An Internet search (Google scholar and MedLine) with the words 'hyperkalemia'/'acute renal failure'/'COX-2 inhibitors'/'adverse drug reactions' and 'etoricoxib' did not yield any results, making our case possibly the first such in the literature.

\section{Conclusion}

Even a short duration of treatment with the new COX-2 inhibitor etoricoxib may have the potential to precipitate renal failure and life-threatening hyperkalemia when administered to selected patients. The importance of considering a patient's existing diet, medications and systemic status before writing a new prescription that may interfere with the potassium physiology cannot be overstressed.

\section{Consent}

Written informed consent was obtained from the patient (or the patient's relatives) for publication of this case report.

Competing interests The authors declare that they have no competing interests.

Authors contributions S.T. was involved in clinical management of the patient and in the manuscript preparation.

S.D. was involved in manuscript reviewing and editing apart from clinical patient care.

\section{Reference}

1. Perazella MA (2000) Drug induced hyperkalemia: old culprits and new offenders. Am J Med 109:307-314

2. Acker CG, Johnson JP, Palevsky PM, Greenberg A (1998) Hyperkalemia in hospitalized patients. Arch Int Med 158:917-924

3. Harris RC (2002) Cyclooxygenase-2 inhibition and renal physiology. Am J Cardiol 89(Suppl):10D-17D

4. Lam Q, Schneider HG (2005) Hyperkalaemia with cyclooxygenase-2 inhibition and hypoaldosteronism. Intern Med J 35(9):572-573

5. Takemoto JK, Reynolds JK, Remsberg CM, Takemoto JK, Reynolds JK, Remsberg CM, Vega-Villa KR, Davies NM (2008) Clinical pharmacokinetic and pharmacodynamic profile of etoricoxib. Clin Pharmacokinet 47:703-720

6. Krumholz HM, Ross SJ, Presler AH, Egilman DS (2007) What have we learnt from Vioxx? BMJ 334:120-123

7. Young D (2007) Few options available for arthritis pain refief. FDA declines to approve Merc's Arcoxia. Am J Health Syst Pharm 64:1020-1022

8. Sharma JN, Jawad NM (2005) Adverse effects of COX-2 inhibitors. Scientific World Journal 5:629-645

9. Akinbamowo AO, Salzberg DJ, Weir MR (2008) Renal consequences of prostaglandin inhibition in heart failure. Heart Fail Clin 4(4):505-510

10. Omalhassan A, Yahaya H, Sarriff A (2009) Incidence of risk factor for developing hyperkalemia when using ACE inhibitors in cardiovascular diseases. Pharm World Sci 31:387-393

11. Zhang J, Ding EL, Song Y (2006) Adverse effects of cyclooxygenase 2 inhibitors on renal and arrhythmia events: metaanalysis of randomised trials. JAMA 296:1619-1632

12. Perazella MA, Tray K (2001) Selective cyclooxygenase-2 inhibitors: a pattern of nephrotoxicity similar to traditional nonsteroidal anti-inflammatory drugs. Am J Med 111:64-67

13. Cannon CP, Curtis SP, FitzGerald GA et al (2006) Cardiovascular outcomes with etoricoxib and diclofenac in patients with osteoarthritis and rheumatoid arthritis in the Multinational Etoricoxib and Diclofenac Arthritis Long-term (MEDAL) programme: a randomised comparison. Lancet 368:1771-1781

14. Braden G, O'shea MH, Mulhern JG, Germain MJ (2004) Acute renal failure and hyperkalaemia associated with cyclooxygenase-2 inhibitors. Nephrol Dial Transplant 19:1149-1153

15. Harris RC, Breyer MD (2006) Update on cycloxygenase-2 inhibitors. Clin J Am Soc Nephrol 1:236-245 